\title{
NIC 1: Presentación de estados financieros
}

Fernández-Pomalaza, Wendy ${ }^{1}$; De la Cruz-Rodríguez, Keyla ${ }^{2}$; Vergara-Durand, Carla ${ }^{3}$; Gonzales-Flores, Sisley

1,2,3,4 Universidad Peruana Union (UPeU), Perú

Recibido 20 de julio de 2014 - Aceptado el 18 de setiembre del 2014

\section{Resumen}

La NIC 1, "Presentación de estados financieros", como una de las Normas Internacionales de Contabilidad, está dirigida a establecer las bases para una buena presentación de estados financieros de un ente económico, de forma exacta y oportuna. La redacción de esta norma la realiź la IASC, actualmente conocida como IASB, en 1997, sufriendo hasta la actualidad diversas modificaciones y remplazando a su
vez a la NIC 1, 5, 13. El Perú está comprometido a generalizar la práctica de esta norma por ser miembro de la IFAC. Esta NIC cumple, además, la finalidad de suministrar información acerca de la situación financiera destinada a los diferentes campos. Asimismo, esta presentación cumple diversos requisitos dados por los principios contables y por algunas NIIF, revelando de esa manera una presentación presentan aspectos de la situación económica, financiera y administrativa de una empresa, mediante el estado de situación financiera que muestra en forma clara el valor de las propiedades, derechos, obligaciones y capital valuados y elaborados, de acuerdo con los P.C.G.A., como resultado de las cuentas reales, estado de resultado en el período integral que presenta información relativa a los ingresos, costos y gastos de una empresa en un tiempo determinado, estado de cambios en el patrimonio que informa de forma detallada los aportes de los socios y la distribución de las utilidades obtenidas en un período específico y, por último, el estado de flujo de efectivo, que consigna el rigen y uso del efectivo de una empresa.

Palabras clave: Estados financieros, oportuno, comparable, razonable y uniforme.

\section{Abstract}

IAS 1, "Presentation of financial statements", as one of the International Accounting Standards, is intended to form a basis for a good IASC, now known as IASB in 1997, to the present suffering various modifications and replacing turn to IAS $1,5,13$. Peru is committed to generalize the practice of this standard as a member of IFAC. This NIC also fulfills the purpose of providing information about the financial position intended for different fields. Also, this presentation serves several requirements given by the accounting and some IFRS, thereby revealing a fair presentation, comparable and uniform. Besides the relevance of IAS 1 , is given by the financial statements, which are tables showing aspects of economic, financial and administrative management of a company, by the statement of financial position showing clearly the value of the properties, rights, obligations and equity valued and developed, in accordance with GAAP as a
result of actual accounts, statement of comprehensive income in the period that presents information on revenues, costs and expenses of a business in a given time, state changes in equity reports in detail the contributions of the partners and the distribution of profits over a specified period and, finally, the cash flow statement, which records the source and use of cash of a company.

Keywords: Financial statements, timely, comparable, reasonable and consisten

\section{Introducción}

La Norma Internacional de Contabilidad 1 "Presentación de estados financieros" se caracteriza, actualmente, por ser una norma que establece bases para una correcta perento presentación uniforme y dado en un monto oportuno; además es una norma internacional que rige la contabilidad en el mundo actual por ser adoptada por las NIIF. Asimismo, comprende principios contabs y estados financieros, los cuales se aplican en la elaboración de su presentación. En este contexto, vemos que la Norma Internacional de Contabilidad 1, cumple un rol importante en la preparación y presentación de estados financieros y, por ello, todo ámbito empresarial debe respetar su aplicación, pues es el medio que ayuda en la toma de decisiones, influyendo en el funcionamiento de la organización.

Este trabajo tiene el propósito de dar a conocer el surgimiento y la finalidad por la cual se realizó la presente NIC. Asimismo, revelar cómo se preparan y presentan los estados financieros.
El primer lugar, se aborda su surgimiento a partir de las modificaciones realizadas hasta la actualidad, además muestra la importancia que cumplen en diferentes campos y la finalidad para diversas personas naturales. También se desarrolla las características a tener en cuenta respecto a la presentación dichos estados, considerando los principios y partidas.

Posteriormente se expone la importancia de los cuatro estados financieros, su forma de presentar y en qué consiste cada uno de ellos. A simismo, se da a conocer que esta no es la única norma que regula los estados financieros, pues existen otras NIC's y algunas NIIF, que norman este instrumento financiero. Por lo tanto, no se han considerado otros aspectos que planteen detalladamente el estado de flujo de efectivo, pues este
tiene específicamente una Norma Internacional de Contabilidad que lo rige.

El presente trabajo es una compilación de datos obtenidos, a traves de una labor en conjunto con la inalidad de ofrecer al lector un texto de referencia, sin pretender que sea un completo y exhaustivo análisis del tema propuesto, sin embargo, se espera que pueda servir

\section{Generalidades}

\section{Antecedentes}

Los antecedentes de Ia NIC 1 son abordados a través de los siguientes aspectos: el origen, sus afirmaciones y sus modificaciones.

\section{Origen}

Como parte del organismo de las normas contables que rigen las actividades de las empresas, relacionadas a la contabilidad, la aparición de la NIC 1 sugiere tres contextos: la emision de las NIC's de manera general, el surgimiento de la NIC de turno, asi como su aplicación, su proceso de revisión y su aprobación en el ámbito nacional.

Las NIC's fueron promulgadas de manera general por la F 1977, cuya misión es servir a interés público, fortalecer la población contable en el mundo y contribuir al desarrollo de economías internacionales fuertes, estableciendo y promoviendo la adherencia a normas cada vez más profesionales y de calidad. Favoreciendo, de esa manera, la convergencia internacional de tales normas y expresando temas de interés público, donde la experiencia de la profesión es más relevante (Junta de Decanos de Colegios de Contadores Públicos del Perú, 2008). A simismo, los organismos que rigen a la IFAC (personal y voluntarios) están comprometidos con los valores de integridad, transparencia y experiencia, siendo también el propósito de la IFAC reforzar la adherencia
de estos valores por parte de los contadores profesionales, los mismos que están reflejados en el Código de Ética para Contadores Profesionales de IFAC (Junta de Decanos de Colegios de Contadores Públicos del Perú, 2008). La IFAC está compuesta por 172 miembros y asociados, en 129 países y jurisdicciones, 10 que representa aproximadamente 2,5 millones de contadores en la práctica pública, la educación, la administración pública, la industria y el comercio (IFAC, 2013); además está compuesto por 163 organismos (Junta de Decanos de Colegios de Contadores Públicos del Perú, 2008); sin embargo, la IASC es responsable de su redacción y aprobación, con autonomía propia, desde el 29 de junio de 1973, conformado por miembros de más de trece paises (Flores, 2002). Cabe aclarar que la como IA SB a partir del 2001 asimismo, este organismo ha emitido y publicado 41 normas internacionales de contabilidad de las cuales se encuentran vigentes 29 : además, ha emitido 8 normas internacionales de información financiera (A paza, 2009).

De acuerdo a lo anterior, según (Ferrer, 2012) la IA SC aprueba y propone el marco conceptual de la primera estructura de la NIC 1, en setiembre de 1997. La emisión de esta norma sustituye, según Mantilla (2011), a dos normas: la NIC 1, llamada Revelación de Políticas de Contabilidad (emitida en 1974) y la NIC 13, llamada Presentación de Activos Corriente y Pasivos Corrientes (emitida en 1979). Sin embargo, Paredes, Reátegui y A yala (2011) afirman que, además de sustituir a la NIC 1 y a la NIC 13, también deroga a la NIC 5 : Información a ser Present
1977).

El Perú está comprometido con la práctica de las NIC's, al ser miembro de la IFAC (Zavala, 2003) y el Colegio de Contadores Públicos de Lima tiene la responsabilidad de determinar su aplicación en el país, por eso se viene difundiendo aspectos teóricos para su divulgación y enseñanza (Concha, 1998) y, por ende, importante para la práctica contable, con mucho más énfasis desde Ia aprobación de la Ley 26887 artículo $223^{\circ}$ (Ortega \& Luján, 2006), siendo esta la Ley General de Sociedades que rige nuestro pais, a partir del ejercicio los estados financieros, de conformidad con las normas legales y los Principios Contables Generalmente A ceptados (PCGA), principios que, de acuerdo a la Resolución de la Contaduría Pública de las Naciones N. ${ }^{\circ}$ 013-98-EF/93.01 (Ortega \& Luján, 2006) y emitido por el Consejo Normativo de Contabilidad, comprende las NIC's y en
forma supletoria a los principios de contabilidad arma supletoria a los principios de contabitad 2003). Por lo cual, en el Perú la NIC 1 "Presentación de Estados Financieros" se lleva a cabo mediante la Resolución CNC N. ㅇ 016-99-EF/93.01 (24.07.99), vigente a partir del comienzo del año 2000 (V era 2007). 


\section{Afirmaciones}

Los estados financieros se pueden afirmar o definir mediante las siguientes fuentes: según autores, glosarios, entidades y la NIC que lo rige.

Según las interpretaciones de los autores, se tiene que los estados financieros vienen a ser una gran gama de información disenada para objetivos específicos (M artínez A., 2011), siendo un conjunto de estados que presentan la situación económica, financiera y administrativa de la empresa (Giraldo, 1996), pues son verdaderos cuadros sinopticos en que la parte numérica como resultado de resúmenes esquemáticos que incluyen cifras, rubros y clasificaciones (García, 2008), siendo a su vez el resultado de coniugar los hechos registrados de la contabilidad, con convenciones contables y juicios personales (M acías, 2011).

También los glosarios definen que estos son aquellos que proveen información respecto a la posición financiera resultados y Estado de Flujos de Efectivo (Glosario del Manual para la Preparación de la Información inanciera, 2008) como cuadros que presentan en forma sistemática y ordenada diversos aspectos de la situación y económica de una empresa (Glosario Tributario, 2008).

A simismo, algunas entidades encargadas de regular la contabilidad en el país, también, 10 definen como documentos contables que muestran la posición y desempeño financiero de una empresa por un período

Por último, la NIC 1 lo afirma, mediante el párrafo 7 , como una representación estructurada de la situación y que pretende cubrir las necesidades de usuarios que no que preten en condiciones de exigir informes a la medida de sus necesidades específicas de información (Paredes, 2008).

Parcialmente, se puede concluir que los Estados Financieros son importantes, tanto en las empresas como en el ente económico, pues proveen información necesaria para adquirir las operaciones realizadas, , para la toma de mejores decisiones.

\section{odificaciones}

a NIC 1, a lo largo de su vigencia, ha tenido varias actualided, IASB ha expuesto tres modificaciones en los años 2003, 2007 y 2011, este último vigente en la actualidad. En abril de 2001, el Consejo de Normas Internacionales interpretaciones emitidas bajo constituciones anteriores continuarían, siendo aplicables a menos y hasta fueran modificadas o aclaradas (Paredes \& A yala, 2009), por lo cual, en 19 de diciembre del 2003 una versión revisada de la N orma Internacional de Contabilidad NIC 1 "Presentación de Estados Financieros" fue emitida por la IASB reemplazando a la NIC 1 y revisada en 1997, (Paredes \& A yala, 2009) por 14 miembros, siendo estos los siguientes (IASB, 2004): Sir David Tweedie (Presidente), Thomas E. Jones (Vicepresidente), M ary E. Barth, Hans-Georg Bruns, Anthony T. Cope, Robert Garnett, Gilbert Gélard, James J. Leisenring, Warren J. T. Smith, Geoffrey Whittingten y Tatsumi Y Y amada.

Luego fue oficializada en el Perú, mediante Resolución . 034-2005-EF/93.01 del Consejo Normativo de Contabilidad, el 2 de marzo del 2005, vigente a partir del $1^{\circ}$ de enero del 2006 (A paza \& Estela, 2006). Las modificaciones contenidas en este apéndice, en incorporadas con la norma revisada, han sido (IA SB, 2004)

Posteriormente, en la segunda modificación internacional, la IASB revisa nuevamente la NIC 1 con el objetivo de "mejorar la capacidad de los usuarios de la información financiera para analizar y comparar la información suministrada en los estados financieros (Paredes, 2007), la cual se emitió, según Ayala, el 7 setiembre de 2007. No obstante, según (Paredes, Nueva Presentación de estados financieros, 2008), en la revista Actualidad Empresarial, afirma que se emitió el 6 de setiembre del 2007 confirmando así una incónnita en cuanto a la fecha de emisión o publicación A simismo reemplaza a la NIC 1 emitida en el 2003 (Paredes \& A yala, 2009), siendo el principal objetivo de los cambios efectuados, el requerir que la información de los estados financieros sea agregada sobre la base de características compartidas e introduzca un estado financiero de resultado completo. (Paredes, 2007).

A nivel internacional, la NIC 1 entró en vigencia a partir del $1^{\circ}$ de enero de 2009 en adelante (Paredes, 2007), es por eso que la IASB recomendo su aplicación anticipada, es decir, que pudo ser aplicada desde un ejercicio anterior, esto es, desde el año 2008 (Paredes \& A yala, 2009).

En el Perú fue oficializada mediante Resolución del EF/94. publicada 28 de agosto de 2010 (Orto Pacherres, \& Díaz, 2011), y vigente a partir del $1^{\circ}$ de nero de 2011 (M antilla, 2011), en función a ello, en el Perú, a partir de esta fecha quedará sin efecto la NIC "Presentación de Estados Financieros" (modificada en el
2003) y que rigió a partir del $1^{\circ}$ de enero del 2006. (Ortega, Pacherres, \& Díaz, 2011).

Cabe senalar, en términos generales, que la NIC 1 revisada incorpora un nuevo estado financiero, modificando algunos de los requisitos de la presentación de esos estados, exige información suplementaria en determinadas circunstancias, así como se revisa la Parminología utilizada en los estados financieros (Ortega, Pacherres, \& Díaz, 2011). Siendo modificado el párrafo 8 (Componentes de los Estados Financieros) en el 2003, por el párrafo 10 (conjunto completo de los Estados . a) Balance por Estado de Situación Financiera, b) Cuentas de Resultados por Estado del Resultado Integral por Estado, () Estado de Cambios en el Patrimonio Neto Flujos de Efectivo y $\mathrm{N}$ tas no se han producido cambios Asimismo se ha agregado un componente llamado "Estado de Situación Financiera al principio del primer período comparativo" (Paredes, 2008). La NIC I requiere dicho estado cuando se produce uno de los supuestos siguientes:

- Una entidad aplique una política contable retroactivamente.

- Realice una represión retroactiva de partidas en sus Estados Financieros.

- Cuando reclasifique partidas en sus estados financieros.

También se modificaron los párrafos 38 y 41 , se suprimen los párrafos 39 y 40 y se añaden los párrafos encabezamientos que preceden a los párrafos $38,38 \mathrm{C}$ y $40^{\circ}$ (Unión Europea, 2013).

A simismo, el último cambio se realiza el 16 de junio de 2011, el Consejo de Normas Internacionales de Contabilidad (IASB) publicó una serie de modificaciones de la NIC 1 Presentación de Estados Financieros en cuanto a presentación de las partidas de otro resultado global (en lo sucesivo, "modificaciones de la NIC 1"). EI objetivo de las modificaciones de la NIC 1 es clarificar la presentación del creciente número de partidas de otro resultado global y facilltar, a los usuarios de los estados financieros, la distinción entre aquellas de dichas partidas que pueden reclasificarse posteriomente en resul tados y aquell as otras que no se reclasificarán nunca 作 10 y se añade el párrafo $10 \mathrm{~A}$ eliminándose el parrafo 12. Se y se elimina el párrafo 81 Se añaden párráos 81 y 22, y se elimina el párrafo 81 . Se anaden los párrafos 81 100 y 115 , el encabezamiento del párrafo 97 y se añade el párrafo 139). (Europea, 2012). Y aquí, en el Perú, el Consejo Normativo de Contabilidad lo aprueba el 1 de agosto del 2012 entrando en vigencia a partir del 15 de noviembre por Resolución CNC N051-2012-EF/30.

\section{Importancia}

En el mundo actual, en el que los usuarios reciben la tempo real $y$ en el que dominan las tecnologías de información, no puede concebirse un mundo empresarial en el cual se tomen decisiones sin sistemas de información útil y confiable, que sirvan de sopote para una adecuada estrategia corporativa. Es por lo, que toda entidad requiere de una gama de información diseñada para fines especificos, siendo parte de esta los estados financieros, que cumplen una función diferentes canpos ser preparados y otorgados a los auditoría.

\section{Administrativo}

Los estados financieros tienen gran importancia en el ámbito administrativo al ser presentados de manera clara prover un y cumplir tres propositos significativos: proveer un plano para determinar los resultados de las
finanzas y operaciones, brindar información hacia quienes dirigen los negocios, y juzgar y evaluar el desarrollo del ente.

Los estados financieros, junto con los informes detall lados suplementarios de las operaciones internas y estadisticas de la gerencia, proveen a la administración un "plano", porque de esa manera se puede determinar la fortaleza o debilidad de las finanzas y de las operaciones (Ralph \& Stewart, 1971).

También brindan información, porque estos informes financieros de operación e interpretación son esenciales para quienes dirigen, manejan y controlan los negocios financieros pueden ser utilizados por la gerencia de las siguientes formas (M artínez A, 2010): 1) medir el estado de costo de producción y ventas, 2) determinar la eficiencia relativa de los departamentos, divisiones, procesos y productos, 3) medir la eficiencia y la productividad de las operaciones para la empresa, 4) apreciar las realizaciones de los individuos en quienes se ha delegado autoridad y asignado responsabilidad, 5) determinar si las nuevas normas de dirección y procedimientos deben ponerse en vigor, 6) evaluar el sistema de control interno, 7) controlar las operaciones y efectuar mejoras en las técnicas de operación, 8) realizar decisiones correctas en relación con las finanzas y con todas las fases de las operaciones, 9 ) justificar ante los inversionistas su actuación en futuros planes y presupuestos.

A simismo, los administradores y propietarios la utilizan para juzgar y evaluar el desarrollo de la entidad a la luz de las políticas, metas u objetivos preestablecidos por la gerencia o dirección de la empresa, pues tales informes comparar el pasado de la empresa con presente y, mediante la aplicación de herramientas 0 

elementos de "control", prever y planear el futuro de la
entidad (Romero, 2010), a partir de una gran necesidad de información que contienen los estados financieros para poder cumplir con las responsabilidades de planeamiento, decisión y control, pudiendo elaborar además cualquier otra información que consideren necesario (A yala, 2003).

a información que surge de este subsistema se utiliza sobre todo en la planeación, la organización, la dirección y el control, tanto en la alta dirección como en los diferentes niveles de la empresa, por lo que en ocasiones se le cataloga como información confidencial (M artínez A., 2010). De esta manera, los estados financieros son importantes en el area administrativa, tanto en su diferentes niveles como inmediato en los estados financieros de un negocio se encuentra en su administración, en las personas que están operando el negocio para los propietarios y que son directamente responsables de las finanzas y de las operaciones.

\section{Financiero}

Los estados financieros, en el ámbito financiero, son importantes porque permite especificamente: 1) elaborar presentar los estados financieros y 2 ) brindar información cuantitativa y descriptiva.

Es el encargado de elaborar y presentar los estados financieros, pues es el que ve las transacciones comerciales, financieras, económicas y sus efectos los usuarios externos de la la fintilidad de servir a resultados de sus operaciones y los cambios en su situación financiera en el período contable terminado. Debido a la diversidad de usuarios de esta información accionistas, sistema financiero, proveedores, acreedores y otros), y a las muy diferentes necesidades de cada uno el campo financiero conlleva a ofrecer información de carácter general, sobre la situación de la empresa (M artínez A ., 2010).

Asimismo, brinda información cuantitativa, expresada en unidades monetarias y descriptivas pues muestra la posición y el desempeno financiero de una entidad (Martínez A. , 2010) identificable y cuantificable que afecta a la entidad, estableciendo medios de control que permitan comunicar información cuantitativa y cualitativa, analizada e interpretada para que los diversos interesados tomen decisiones en relación de dicha

Aquí vemos que el campo financiero es indispensable para dar y presentar los Estados Financieros, pues es el período contable, siendo estos claros y precisos.

Auditoría En el campo de auditoría, la importancia radica en que elaborada, para facilitar a los auditores el cumplimiento de los principales objetivos: analizar el informe contable e informar con la máxima garantía la no existencia de errores.

Los auditores analizan el informe contable pues, después de hacer su investigación con las normas y procedimientos generalmente aceptados de auditoria, indican que los estados financieros presentan razonablemente la situación financiera y los resultados de las operaciones conforme a los principios contables por lo general aceptados y aplicados en forma consistente. De esta manera, los estados financieros auditados son de gran registros de contabilidad, por contadores públicos independientes; y a su vez, pos registros contables han sido estudiados por ellos (Ralph \& Stewart, 1971).

La aplicación del análisis de estados, como parte del proceso de auditoría, es indispensable, pues permitirá omisiones (Tan áreas posibles de faltas, errores u una auditoría de estados financieros para determinar si estos, en general, han sido elaborados de acuerdo con el criterio establecido, utilizando la base de efectivo o alguna otra base de contabilidad adecuada para la organización, determinando así si los estados contienen errores importantes u otras declaraciones erróneas (A rens, Elder, \& Beasley, 2007). A simismo, la forma más común de que los usuarios obtengan información confiable es realizar una auditoría independiente, pues solo asi proporciona seguridad a los usuarios; pero si al incorrectos, tanto los usuarios como la administración pueden demandar al autor (A rens, Elder, \& Bensley, 2007). Es por eso que los auditores tienen una responsabilidad legal considerable por su trabajo.

A medida que el nivel de complejidad de los negocios aumenta, ya no es suficiente que los auditores se enfoquen solo en las transacciones contables, pues una visión integrada de la auditoría considera tanto el riesgo de errores como los controles de operación pensados para prevenir errores, siendo cada vez más una perspectiva estratégica de la entidad empresarial (A rens, Elder, \& B easley, 2007).

Los auditores, a fin de emitir un dictamen, también analizan los estados financieros. El resultado final de la auditoría es un dictamen sobre la razonabilidad de la sresentación de estados financieros que explican la de una empresa, por lo cual, el objetivo principal del auditor es obtener la máxima ganancia posible sobre la no existencia de errores e irregularidades, intencionados o no, que afecten la veracidad de los estados financieros, ya que es indispensable, pues permitirá determinar las áreas posibles de faltas, errores u omisiones (Tanaka,
2005). Cabe señalar que los estados financieros deben ser bien elaborados, porque los auditores se encargan de revisar, analizar y ver que la información congruente con la realidad garantice el éxito de una empresa, libre de cualquier manejo indebido.

Como vemos, los estados financieros son preparados con el objetivo de presentar un informe acerca de la situación desarrollo financiero en los diferentes campos laborales, como consecuencia de las operaciones realizadas. Para eso, es necesario saber que la bondad de los juicios depende de la competencia e integridad de quienes lo aplican y de su apego a los principios de contabilidad (Heffes, Holguin, \& Galan, 1994).

\section{Finalidad}

a finalidad de la NIC 1, "Presentación de Estados situación financiera (A paza \& Estela, 2006), establece bases para su presentacion, a fin de asegurar comparabilidad (Vera, 2007). A demás establece requerimientos generales para tal, la estructura y requerimientos mínimos del contenido de los estados financieros (M antilla, 2011), asimismo, (Ferrer, 2012) agrega que su finalidad es suministrar información clara y mostrar los resultados de la gestion realizada, por los administradores, con los recursos que se les ha sido confiados. Por ello, aportan información sobre activos, pasivos, patrimonio neto, ingresos, gastos, resultado y flujos de efectivo. $Y$, al realizar los resultados, los estados financieros presentan la información y ayudan a los diferentes usuarios dependiendo de su realidad, accionistas, gerentes y empleados o trabajadores.

\section{Accionistas}

Estos son los que proporcionan recursos a la entidad, (Romero, 2010) por lo cual tienen mayor exigencia en obtener la información, por tanto, sus intereses son más amplios, puesto que su participación está afectada por motivos:

El primero refiere al interés en la inversión del capital, anal izando e interpretando datos. Están interesados en la inversión del capital y en los activos, pues son ellos los que disponen de los recursos como menciona Romero, arriba, además velan por sus intereses, puesto que su participacion está con picionilida por to pus los afectos. (Tanaka, 2005).

Analizan e interpretan datos presentados, pues los necesitan en la decisión si deben comprar, vender o Tha dividendos (Tanaka, 2005).
Gerentes

Los gerentes utilizan las herramientas de estados financieros con dos propósitos: la finalidad y ventajas. La finalidad es ejercer control sobre la empresa y verla de la misma manera que la ven importantes agentes fuera de ella como son la SUNAT, las instituciones financieras, entre otras (Tanaka, 2005), esto si la presentación de los estados financieros están correctos, claros, precisos y entendibles.

Proporciona ventajas al realizar un seguimiento de los datos financieros y las relaciones básicas las cuales son: determinan las transacciones de la empresa, evita que los gerentes no se pierdan en el laberinto de situaciones que se generan dla a dia en una empresa y permite observar que tiene la empresa.

\section{Trabajadores}

Para referirse al ámbito de los trabajadores, es necesario tomar en cuenta: su importancia e interés en obtener información acerca de su estabilidad y rentabilidad.

M uchos no toman en cuenta la importancia y función de los trabajadores en el ámbito donde laboran, sin
embargo, la Constitución Política de 1993, en su A rt. 56 (Tribunal constitucional del Perú, 1993), contempla el derecho de los trabajadores a participar en la gestión y utilidad de la empresa donde laboran, de acuerdo con la modalidad de esta. Y, en cuanto a la participación en la propiedad, la constitución establece que los trabajadores participan en la propiedad de las empresas cuya propicio referirse a los sistemas de participación ase actualmente existe en nuestro sistema laboral, es decir a los mecanismos de los derechos de participación de los trabajadores en la empresa sea en forma individual 0 colectiva, pero no a través del trabajo normal para el cual fueron contratados, sino, de la misma forma que los socios, tomando parte de las decisiones, obteniendo porcentajes del capital social, y también, de las utilidades generadas en el ejercicio.

Los empleados y sus agrupaciones representativas se encuentran interesados en obtener informacion acerca de la estabilidad y la rentabilidad como empleados, información que les ayude a conocer la capacidad de la empresa para pagar adecuadamente las remuneraciones, los beneficios de jubilación, obtener sueldos más altos, trabajar menos horas, disfrutar de mejores condiciones de trabajo, contar con seguro de vida, así como el específicamente, los empleados están interesados en los estados financieros para determinar el progreso de la compañía y los resultados de la explotación del negocio, durante el año, y las perspectivas para los años venideros. Los estados financieros combinados con otros 
informes suministrados por el negocio podrán reflejar los problemas económicos que afectan a la dirección, a los empleados y a los accionistas (Kennedy \& M cM ullen, 1978). Por ese motivo, los estados financieros necesitan estar bien elaborados para cumplir las finalidades de los trabajadores, y asi poder beneficiar a la empresa, ya que estando estos bien capacitados e informados sobre su rentabilidad, podrán ejercer una mayor eficiencia en su campo laboral, pues nestra Constitución ha consagrado el derecho de los trabajadores a participar en las

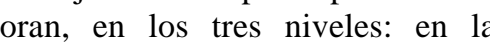
propiedad, en la gestión y en las utilidades.

\section{Características generales}

\section{Presentación razonable}

os estados financieros deben presentar razonable y fidedignamente la situación y el rendimiento financiero conforme a la aplicación de las NIIF, para lo cual toma en consideración tres criterios: compensación, frecuencia de información y comparabilidad, asimismo, uniformidad en la presentación.

\section{Compensación}

Este punto plantea esencialmente dos contextos: cambios producidos a lo largo de su aplicacion y compensación de partidas.

a NIIF, desde su aparición y cambio que suscitó en el 2003 a nivel mundial, modifica el criterio de compensación, especialmente la del párrafo 32 , puesto ya no la NIC (IFRS, 2008), asimismo, dentro de las partidas distintas a las ordinarias, se eliminan el ejemplo de las partidas extraordinarias que ya no existen, de acuerdo con la actual normativa contable (Ortega Luján, 2006).

La compensación de partidas establece que no se compensará activos con pasivos, salvo cuando ello esté exigido o permitido por alguna NIIF, de la misma forma se tratará las partidas de ingresos y gastos, la cual se podrá compensar siempre y cuando genere la misma operación, y con ello refleje la sustancia de dicha operación (A paza \& Estela, 2006), además de las ganancias, pérdidas y gastos relacionados que provengan de transacciones 0 hechos similares que no sean significativos (V era, 2007). Al respecto a esto, (Sinisterra, Polanco Izquierdo,
algunos ejemplos significativos:

Las pérdidas y ganancias por la venta de activos no circulantes.

Los gastos que hayan sido reembolsados a la

empresa, como consecuencia de un acuerdo

contractual con terceros.
Figura 1. Compensación de partidas

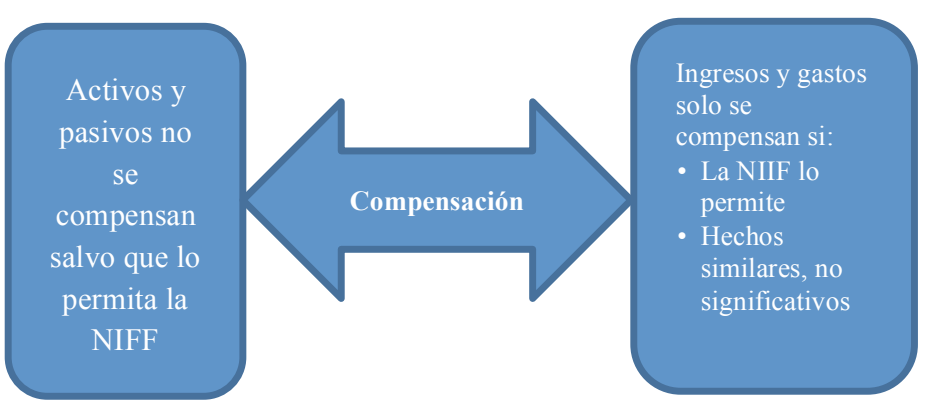

Fuente: Elaboración propia

Sin embargo, se debe tener en cuenta que para reflejar el fondo de la transacción se puede efectuar una deben reflejar la imagen fiel de las transacciones de la misma (Gómez, 2005).

Frecuencia de información y comparabilidad.

Al presentar los estados financieros, se toma

también en consideración los siguientes

criterios: frecuencia de información y comparabilidad.

La información, revelada por los estados financieros, debe mantener una frecuencia y un período contable de un ano, pues normalmente una entidad prepara, de forma comprenden un período, anul. No obstante a entidades prefieren informar, por razones prácticas, sobre períodos de 52 semanas, la cual esta norma no prohíbe esta práctica (IFRS, 2008). Sin embargo, cuando una entidad cambia el cierre del período sobre el que informa y presenta los estados financieros en un período contable, superior 0 inferior a un año revela, además del período cubierto por los estados financieros: la razón para utilizar un período de duración inferior o superior y el hecho de que los importes presentados en los estados financieros no son totalmente comparables (Ferrer, 2012).

La comparabilidad de la información, entre períodos, ayuda a los usuarios en la toma de decisiones económicas, sobre todo al permitir la evaluación de tendencias en la información financiera con propósitos predictivos (IFRS, 2008), por lo cual se presenta tres período anterior, aquello que se presenta cuando comparación resulte desigual y sus ventajas. La presentación de información que un ente económico
revela debe ser comparativa, con respecto al período revela debe ser comparativa, con respecto al periodo los estados financieros y sus notas relacionadas (Paredes, 2007), también deben revelar con todo lo que está incluido en los estados financieros del ejercicio en turno (Paredes, 2009). La información debe ser de tipo descriptivo y narrativo (Sinisterra, Polanco Izquierdo, \& Henao, 2011), a menos que sea cambiada o porque la NIIF lo permita o requiera otra cosa. (IFRS, 2008). A demás, (Ferrer, 2012) agrega que la información de una empresa es comparable a través del tiempo, la cual se financieros sobre bases uniformes.

Si se efectúan cambios de presentación o reclasificación de los estados financieros, se deberá modificar los importes comparativos del ejercicio anterior (Gomez, 2005) y revelar (Paredes, 2009): 1) la razón para no reclasificar los importes y 2) la razón para no reclasificar la naturaleza de los ajustes que tendrían que haberse efectuado si los importes hubiesen sido reclasificados (Sinisterra, Polanco lzquierdo \& Henao, 2011): 1) la naturaleza de la reclasificación 2) el importe de cada uno de los elementos o de clase de elementos que fue eclasificada y 3) lajust opta por reclasificar.

Esto revela que su principal ventaja es mejorar la comparabilidad de la información entre ejercicios o períodos que ayudan, a los usuarios, en la toma de decisiones economicas, la cual permite también la evaluación de tendencias de información financiera con propositos predictivos (FRS, 2008), y ayuda a que la información sea útil en el analisis de los estados financieros de una entidad. (Paredes, 2009).

A sí se llega a la conclusión de que la presentación de estados financieros comparativos, en informes anuales y de otro tipo, realza la utilidad de dichos informes y cambios actuales que afectan a las enpresas. presentación también subraya el hecho de que los estados referentes a una serie de períndos resultan mucho más relevantes que los que abarcan uno solo, y que las
cuentas de un período son únicamente un anticipo de lo (Merrisey, 1977).

\section{Uniformidad en la presentación}

La presentación y clasificación de las partidas en los estados financieros debe ser conservada de un período a otro (A paza \& Estela, 2006), es decir deben mantener una uniformidad; pero si se realiza lo contrario, como consecuencia ocurrirán algunos cambios en lo presentado. Es por eso que se plantea: las razones que justifican los cambios y al gunos ejemplos o casos.

Las razones que justifican los cambios son: 1) cuando se roduce un cambio en la naturaleza de las operaciones de la empresa, que demuestra que el cambio dará lugar a una presentación más apropiada (A paza \& Estela, 2006) u otra clasificación, tomando en consideración los criterios para la selección y la aplicación de políticas contables. (Paredes, 2009) y 2) estar obligado a realizar interpretacion (A paza \& Estela, 2006) que requiera un cambio en la presentación (Estela, 2009).

Algunos casos se constituyen ejemplo de los posibles cambios en los estados financieros:

En una adquisición o disposición significativa, 0 una revisión de la presentación de los estados financieros, que podrían sugerir que dichos estados financieros necesitan ser presentados de forma diferente (IFRS, 2008).

Si se realiza un cambio de presentación, y dicho cambio proporciona información fiable y más relevante para los usuarios de los estados financieros. (Paredes, 2009).

Cuando se produce algún cambio y, con el fin de preservar la comparabilidad de la información del año actual, cambia los importes correspondientes a la información del año procedente, a menos que resulte imposible hacerlo (A paza \& Estela, 2006)

\section{Principios contables}

La NIC 1 establece que los estados financieros se elaboran siguiendo los principios contables generalmente aceptados, siendo los más significativos por su empleo:

\section{Negocio en marcha}

Al elaborar los estados financieros, la gerencia evaluará la capacidad que tiene una entidad para continuar en funcionamiento, es por ello que elabora la información bajo la hipotesis de negocio en marcha, la cual se reduce a los siguientes puntos: la importancia de la gestión de la empresa y la no existencia de una alternativa realista.

Tal como lo señala el marco conceptual como postulado hipótesis de lompresa en funcionamiento (Góm pues al elaborarlos, la gerencia evaluará la capacidad que tiene una entidad para continuar en funcionamiento, a menos que la gestión pretenda liquidar la entidad o cesar menos que la gestion pretenda liquidar la entidad o cesar
en su actividad (IFRS, 2008). Y en el caso de que la gestión crea que existen circunstancias que provean duda sobre la posibilidad de que la entidad siga en funcionamiento, normalmente se procederá a revelarlas en los estados financieros (Paredes, 2009).

Una entidad elabora, también, los estados financieros bajo la hipotesis de negocio en marcha, a menos que no exista otra alternativa más realista de proceder, pues cuando la gerencia, al realizar esta evaluación, es consciente de la existencia de incertidumbres importantes, relativas a eventos o condiciones que pue au apotar didas significativas cobre la posibilidad 

procederá a revelarlas, también, en los estados
financieros (Ferrer, 2012). Y cuando una entidad no prepare los estados financieros bajo la hipótesis de negocio en marcha, revelará ese hecho junto con las hipótesis sobre las que han sido elaborados y las razones por las que la entidad no se considera como un negocio en marcha (Gómez, 2005). A simismo, si hubiera incertidumbre en cuanto a la continuidad de la empresa, ello deberá revelarse con los planes de acción, de ser el caso (Paredes, 2009).

Es por ello que, para evaluar la continuidad de una empresa, debe tenerse en cuenta aspectos que se señal

Tendencias negativas como: pérdidas recu-

rentes, deficiencias de capital de trabajo, flujos de efectivo negativo, etc.

Señales de posibles dificultades financieras como: incumplimiento de obligaciones, problemas de acceso al crédito, refinanciaciones, venta de activos importantes, etc.

Otras situaciones internas o externas tales como: restricciones jurídicas a la posibilidad de operar, huelgas prolongadas, catástrofes naturales, cambios tecnológicos y otro que resulte pertinente.

A simismo, al evaluar también si la hipótesis de negocio en marcha resulta inapropiada, la gerencia tendrá en quenta todá la ins mación disponible sobre el futuro, partir del final de período sobre el que se informa, sin

\section{Devengado}

Este principio, base en la elaboración y presentación de los estados financieros, también llamado base contable de acumulación se plantea su comprensión bajo los la elaboración de tales estados financieros.

Las variaciones patrimoniales, que se deben considerar para establecer el resultado económico, son las que corresponden a un ejercicio, sin entrar a distinguir si se han cobrado o pagado durante dicho período (Tanaka, 2005); entonces, como se puede comprobar el principio de devengado, es un principio muy importante para el registro de las operaciones dentro de la empresa, ya que el período que le corresponde Además el postulado básico de la devengación contable, actualmente incluye y define los conceptos siguientes: transacciones, transformaciones internas, otros eventos, en su totalidad, momento en que ocurren, realizados y período contable (Romero, 2010). Al aplicar este principio, en la elaboración de estados excepto sobre información de flujos de efectivos (Paredes, 2009), por lo cual el ente económico el abora sus estados financieros, utilizando la hipótesis contable: acumulación o devengado, por lo cual reconoce las partidas de activos, pasivos, ingresos y gastos, cuando satisfaga las definiciones y los criterios de reconocimiento previstos en el marco conceptual para tales elementos (Estela, 2009). A demás, se agrega que los gastos se reconocen en el estado de resultados sobre la base de una asociación directa entre los costos incurridos y la obtención de las partidas correspondientes de ingresos. Esto, al ser presentado, informan a los usuarios no solo de las transacciones pasadas relativas al pago y pagar en el futuro y de los recursos que presenta efectivo que están por ser recibidos en el futuro lo cual es útil en la toma de decisiones económicas (Romero, 2010).

\section{M aterialidad o importancia relativa}

Este principio se refiere a que muchas veces los gastos, en una empresa, pueden agruparse cuando el valor que representan no es muy significativo. Tambien ha de considerarse que el concepto de materialidad 0 importancia relativa está por encima de la aplicación de cualquier norma o requerimiento, por lo cual se debe analizar el caso y no actuar mecanicamente. La NIC de turno plantea tres formas de utilizar este principio al preparar los estados financieros: presentacion separada de partidas, agrupación de partidas y partidas irrelevantes.

Un ente económico debe presentar de forma separada cada clase de partidas similares que tenga importancia que no sean materiales (Estela 2009) o no cumplan el requisito de importancia relativa (Paredes, 2009). A demás, la materialidad dependerá de la magnitud y la naturaleza de la omisión o inexactitud, enjuiciada en función de las circunstancias particulares en que se haya producido, pues una combinación de ambas, podría ser el factor determinante (Paredes \& A yala, 2009).

Los estados financieros son el producto que se obtiene del procesamiento de un gran numero de transacciones $y$ otros sucesos que se agrupan por clases, de acuerdo con su naturaleza o función (Paredes, 2009), a esto se le considera agrupación de partidas. I gualmente de acuerdo, la etapa final del proceso de agregación y clasificación es constituirán el contenido de los estados financieros (IFRS, 2008), es decir si una partida concreta no fuese material o no tuviera importancia relativa por sí sola, se agrega a otras partidas, ya sea en los estados financieros 0 en las notas. Por ello, una partida que no tenga la suficiente materialidad o importancia rel ativa como para requerir presentación separada en estos estados inancieros, puede requerir presentación separada en las notas (Paredes, 2009).

También no se requiere información a revelar, si la partida no es importante, por eso una entidad no necesita revelar una información específica requerida por una NIIF, si esa información carece de importancia relativa (IFRS, 2008)

\section{Elementos o partidas}

\section{activos}

Son conjuntos de bienes, derechos y valores que cuenta la empresa, ya sea a corto a largo plazo (Giraldo, 1996). los bienes y erechos tangibles a intangibles de propiedad del ente económico, de los cuales se espera tener beneficios presentes o futuros que en la epresentación de su utilización, son fuentes potenciales de beneficios (Rincón, 2009). Pues son recursos obtenidos por una organización en el pasado, de los cuales se espera generar ingresos en el futuro (Sinisterra, Polanco Izquierdo, \& Henao, 2011) siendo un recurso que controla a la empresa como resultado de sucesos pasados, previendose que los beneficios económicos futuros de ese recurso fluyan a la empresa (Flores, 2002). Asimismo, se clasifica en dos tipos: activos corrientes y activos no corrientes.

Los activos corriente vienen a ser los bienes, valores y erechos que posee la empresa en un período de ejercicio contable, es decir en doce meses (Giraldo, 1996). Está representado por el efectivo y equivalente de efectivo, realizables, activos biológicos, sin embargo, la NIC de turno menciona cómo clasificar estos activos, explicando que esto sucede cuando: 1) se espera realizar ex activo 0 tiene la intención de venderlo o consumirlo, en su ciclo normal de operación. 2) se mantiene el activo principal mente con fines de negociación y 3) el activo es efectivo 0 equivalente de efectivo a menos que este se encuentre restringido y no pueda ser intercambiado ni utilizado para cancelar un pasivo por un ejercicio del mismo.

Los activos no corrientes son bienes, valores y derechos que tiene la empresa en más de un año (Giraldo, 1996). Está representado por inversiones financieras, derechos por recuperar, activos realizables, activos biologicos, inversiones inmobiliarias, inmuebles, maquinaria y equipo, activos intangibles y otros activos cuya realizablecido como corriente (Estela, 2009).
Son un conjunto de deudas, obligaciones y compromisos que tiene la empresa con segundas y terceras personas a corto y largo plazo. También agrupa el conjunto de cuentas que representan las obligaciones contráidas por el ente económico, en desarrollo del giro ordinario de su actividad, pagaderas en dinero, bienes o en servicios
(Rincón, 2009). Esta también se divide en dos tipos:
pasivo corriente y pasivo no corriente: pasivo corriente y pasivo no corriente

El pasivo corriente es aquel integrado por deudas y obligaciones a cargo de una entidad económica cuya exigencia 0 vencimiento se da dentro del próximo año dentro a partir de la fecha de estado de resultado, o bien, Romero corriente cuando: 1) se espera liquidar en el curso normal de la operación de la empresa y 2) debe liquidarse dentro del período de doce meses, desde la fecha del balance.

Según la NIC 1 "Presentación de Estados Financieros" una entidad clasificará un pasivo como corriente cuando: 1) se espera liquidar el pasivo en su ciclo normal de operación, 2) se mantiene el pasivo principalmente con fines de negociación, 3) el pasivo debe liquidarse dentro de los doce meses siguientes a la fecha del período sobre el que se informa 04 ) no tiene un derecho incondicional para aplazar la cancelación del pasivo durante, al menos, los doce meses siguientes. Tambien menciona que algunos pasivos corrientes, tales como las cuentas comerciales por pagar y otros pasivos acumulados (devengados), ya sea por costos de personal o por otros costos de operación, integran el capital de trabajo Una entidad clasifica estas partidas de de la entidad. pasivos corrientes, aunque se vayan a liquidar después de los doce meses de la fecha del período sobre el que se informa.

Los pasivos no corrientes son los que no cumplen con los requisitos de los pasivos corrientes mencionados anteriormente, puesto que están representados por todas plazo establecido como corrientes (Estela, 2009).

\section{Patrimonio}

Para definir patrimonio, se tiene en consideración tres ideas claves: obligaciones contraídas con socios, activo neto de una entidad y al activo residual.

Se dice que son obligaciones con socios o accionistas de la empresa, porque los pasivos han sido suministrados ordinario del negocio y no del ente económico, sino de los socios. (Rincón, 2009). 
Desde el punto de vista económico, el capital representa e valor que contablemente tienen para la entidad sus activos y pasivos sujetos de reconocimiento en los estados financieros: por esta razón se le conoce también como activo neto de una entidad (Romero, 2010).

Asimismo, es la parte residual de los activos de la empresa, pues el resultado de deducir todos los pasivos, (Flores, 2002). Comprende las siguientes partidas: patrimonio atribuible a la matriz, intereses minoritarios, capital, acciones de inversión, capital adicional, resulcion no realizados, excedente de revaluación, valorización de instrumentos financieros, reservas legales, resultados acumulados, diferencias de conversión y otras
reservas (Estela, 2009).

\section{Estructura y contenido (estados)}

\section{Estado de situación financiera}

Llamado anteriormente balance general, es un documento contable que informa en una fecha determinada la situación financiera de la empresa, presentando en forma clara el valor de sus propiedades, derechos, obligaciones y capital, valuados y elaborados de acuerdo con los Principios Contables Generalmente Aceptados, en el cual aparecen como resultado las cuentas reales, y sus valores deben corresponder exactamente a los saldos ajustados del libro mayor y mantiene su presentación en orden decreciente de liquidez del activo y exigibilidad del pasivo (Ferrer, 2012), y muestra la situación financiera de la empresa (Tanaka, 2005). También es definida por el Comité sobre Públicos Titulados como un restado como resultado real o constructivo del cierre de los libros de cuentas que se llevan conforme a los principios contables (Ralph \& Stewart, 1971), por lo cual incluye todos los movimientos susceptibles de ser registrados contablemente, desde la fecha de constitución de la empresa hasta la fecha de su formulación, además de ser emitido por las entidades lucrativas y por las entidades con propósitos no lucrativos (Rojas, 2011). Es por ello, que comprende los siguientes puntos para su presentacion: información a presentar, separación entre partidas corrientes y no corrientes e información a presentar en notas.

\section{Información a presentar}

La información revelada en este estado debe cumplir tres criterios específicos: información mínima a incluirse en los estados financieros, orden de presentación y para la presentación de información adicional.

La información mínima (IFRS, 2008) que se incluye en el estado de situación financiera son partidas que presenten los siguientes importes, tales como: inmuebles,

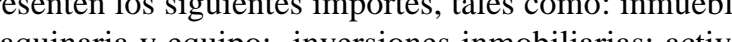
intangibles y activos financieros: inversiones contabilladas utilizando el método de la participación: activos biológicos; existencias; deudores comerciales y otras cuentas a cobrar y efectivo, y otros medios líquidos equivalentes.

Etotal de activos clasificados como disponibles para la venta y los activos incluidos, en los grupos enajenables de elementos, que se hayan clasificado como disponibles para la venta de acuerdo con la NIIF 5 A ctivos no corrientes disponibles para la venta y actividades interrumpidas: acreedores comerciales y otras cuentas a pagar; provisiones; pasivos financieros; pasivos y activos por imptestos corfentes, según se definen en la NIC 12 impuesto sobre las ganancias; pasivos y activos por pasivos incluidos en los grupos enajenables de 12 ic clasificados como disponibles para la venta de acuerdo con la NIIF 5; intereses minoritarios, presentados dentro del patrimonio y el capital emitido y reservas atribuibles a los propietarios de la dominante.

Como podemos ver, estas son rúbricas específicas que contienen importes correspondientes a las partidas ya
nombradas, las cuales se incluyen en el estado de situación financiera (Estela, 2009).

La NIC 1, específicamente, no prescribe un orden de presentación ni un formato concreto de cómo se presentarán dichas partidas, pues solo menciona cuáles son las partidas minimas que deben presentarse, pero sí prescribe cómo se clasifica dichas partidas cuando el se menciona se menciona que los activos y los pasivos se debe Polanco Izquierdo \& Henao, 2011), siendo (sta la única forma de clasificación de partidas en la actualidad (Paredes, 2009), puesto que anteriormente se solía presentar en tres grupos: circulante, fijo y diferido (Rojas, 2011).

A simismo, una entidad decidirá presentar partidas adicionales de forma separada en función de una evaluación a los criterios: la naturaleza y liquidez de los
activos; la función de los activos dentro de la entidad; y activos; la función de los activos dentro de la entidad; y

los importes, la naturaleza y el plazo de los pasivos.

\section{Distinción entre partidas corrientes y no corrientes}

En este punto de presentación del estado de situación financiera, vemos que la NIC de turno también menciona dos aspectos fundamentales de esta distinción, en las presentación de activos y pasivos, en orden de liquidez y base mixta.

Es importante distinguir entre las partidas corriente y no corrientes, porque proporcionará una información útil al distinguir los activos netos que están circulando de los utilizados en las operaciones a largo plazo de la entidad, asimismo, esta distinción servirá también para destacar tanto los activos que se espera realizar en el transcurso del ciclo normal de la explotación, como los pasivos que se deben liquidar en ese mismo período (IFRS, 2008).

Si la presentación de dichas partidas se presenta en orden de liquidez, es decir en orden ascendente (de inmovilizado a efectivo) o descendente (de efectivo a inmovilizado) (A paza, 2011), proporcionará información flable y más relevante que la presentación si está dividida en corriente y no corriente; asimismo, si se para cada activo y pasivo, lo que se espera receuperar cancelar en los doce meses posteriores a la fechar del estado de situación financier (A paza, 2011), asimismo en el caso que sea mixta, es decir presenta algunos de sus activos y pasivos empleando la clasificación corriente-no corriente, y otros en el orden de liquidez se podrá realizar, siempre que esto proporcione información fiable y más relevante (Ortega, Pacherres, \& Díaz, 2011).

\section{Propósitos}

El estado de situación financiera cumple específicamente dos propósitos fundamentales: ofrecer información a usuarios externos y ser fuente de información a gerentes y ejecutivos.

Ofrecer información a usuarios externos de la organización, quienes sin tener acceso a sus registros contables, se interi

A simismo, es fuente de información a gerentes y demás ejecutivos, puesto que interviene en la toma de decisiones financieras, y logra mejores resultados en ente económico (Sinisterra, Polanco Izquierdo, \& Henao, 2011).

\section{E stado del resultado integral del período}

Este estado presenta información relativa a los ingresos, costos y gastos, determinando si la operación de la empresa es adecuada, en un periodo determinado, llamado "periodo contable" (Juárez, 2011), en el cual se agruparán los ingresos y gastos por naturaleza, revelando información sobre los importes de los principales tipos de productos y gastos (A paza, 2011). A simismo, es la Ilave que da paso al aspecto íntimo de la sociedad, pues analiza el resultado obtenido en el ejercicio, y es la registrado durante el año las cargas y los ingresos de empresa Por tanto, así como el estado de situación financiera tiene un carácter estático, ya que presenta una fotografía del estado patrimonial de la empresa el etado de resultado integral de periodo que tiene un carácter dinámico presentando de forma resumida los resultados de operación de la empresa y permitiendo analizar cómo se ha llegado a conseguir la utilidad de aquel determinado período (A paza, 2011). Es por ello que a presentar y resultado del período.

\section{Presentación}

Una entidad tiene dos opciones para presentar su estado de resul tados integral total para un período: en un único estado 0 en dos estados.

En único estado se presentará el estado de resultado integral, la cual debe tomar en cuenta todas las partidas de ingreso y gasto reconocidos en el periodo (Effio, (A paza, 2011).

En dos estados se presentará el estado de resultado y el estado de resultado integral, en cuyo caso el estado de
resultados presentará todas las partidas de ingreso y gasto reconocidas en el período (IFRS, 2008), excepto las que estén reconocidas en el estado integral total fuera del resultado, tales como lo norma la NIIF (A paza, 2011).

\section{Información a presentar}

La información a presentar, en este estado, debe cumplir como minimo los siguientes aspectos: la información minima a presentar, el momento de revelación de partidas por separado y la importancia de revelar partidas
adicionales.

Una entidad, como mínimo, incluirá partida que presente los siguientes importes de período (A paza, 2011): los

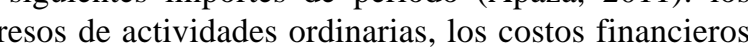
y la participación en el resultado de las inversiones en
asociadas y entidades controladas de forma conjunta contabilizadas, usando un método de la participación.

\section{El gasto por impuestos}

Un único importe que comprenda el total del resultado después de impuestos de las operaciones discontinuadas, y la ganancia o perdida despues de impuestos reconocida en la medición al valor razonable menos costos de venta, o en la disposición de los activos netos que constituyan
la operación cotidiana.

El resultado, si una entidad no tiene partidas de otro resultado integral, no es necesario presentar esta línea. clasificado por naturaleza, excluye los importes a los que hace referencia a continuación: 1) participación en el resto del resultado integral de las asociadas y negocios conjuntos que contabilicen según el método de la participación y 2) resultado integral total. 
Cuando en las partidas de ingreso y gasto sean importantes su naturaleza e e importe, se revelará por presentará un desglose de los gastos, utilizando para ello una clasificación basada en la naturaleza de los mismos o en función que cumplan dentro de la entidad (Estela, 2009). Según el método de clasificación por naturaleza de los gastos, estos se agrupan en el estado de resultado integral, de acuerdo a su naturaleza y no se redi stribuyen entre las diferentes funciones dentro de la entidad (Effio, 2011); y según el método de clasificación por función de los gastos, estos se agrupan de acuerdo con su función, como parte del costo de ventas 0 , por ejemplo, de los costos de actividades de distribución o administración, pues como mínimo una entidad llega a revelar, según otros gastos (A paza, 2011).

Tabla 1. El gasto por impuestos

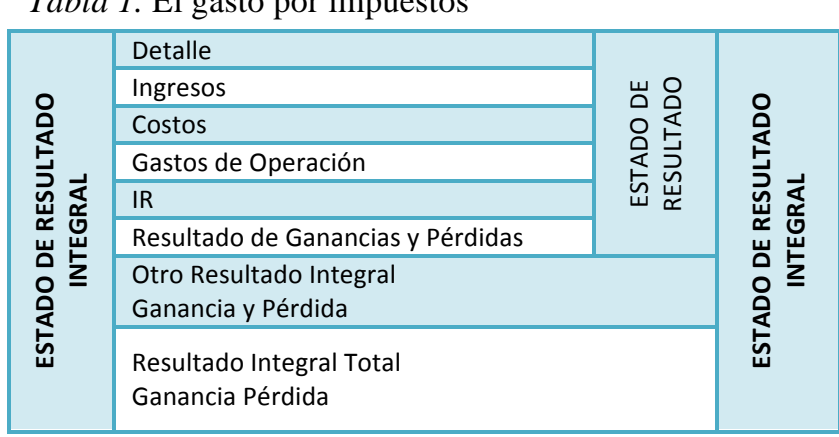

uente (A paza, A nálisis de NIIF para PY M ES y casos prácticos aplicando el PCGE, 2011).

Asimismo, la revelación de partidas adicionales cumple una gran importancia al ser incluidas en el estado de resultado integral, y al ayudar a los usuarios a comprender el rendimiento financiero alcanzado, asi como al realizar proyecciones futuras sobre sí mismo, como resultado de este (Paredes, 2009).

\section{Elementos}

os elementos relacionados con la medición o val oración en este estado son específicamente: ingresos y gastos.

Los ingresos son aumentos en los activos del ente económico provenientes de sus actividades orientadas hacia las utilidades y que origina flujos de efectivo positivos (M eigs, Willims, Hka y Bettner, 2000), por 10 cual, llegan a ser incrementos en los beneficios eercicio en forma de entradas de valor de los activos 0 disminución de los pasivos (Estela, 2009) que, a su vez, producen incrementos en el patrimonio que son de naturaleza diferente a las contribuciones hechas por los socios (Flores, 2008).

Los gastos son disminuciones en los activos de la empresa provenientes de sus actividades dirigidas hacia la obtención de utilidades, que dan como resultado un 政, Bettner, 2000), como disminuciones en los beneficios económicos, producidos a lo largo del ejercicio en forma de salidas del valor de los activos o aumento de los pasivos, lo que, a su vez, dan como resultado disminuciones en el patrimonio. A simismo, estas no estann relacionadas con las distribuciones realizadas a las propiedades de este patrimonio (Flores, 2008), Ilegando a disminuir la utilidad económica de la empresa (Estela, 2009).

\section{Otros estados}

\section{E stado de cambios en el patrimonio}

Este estado se puede definir a partir de tres fuentes: según autores, NIC de turno y la Superintendencia del Comision Nacional Supervisora de Empresas y $\checkmark$ alores (CONACEV).

Según autores, el estado de cambios en el patrimonio muestra, en forma detallada, los aportes de los socios y la distribución de las utilidades obtenidas en el período (Estela, 2009), dando un resultado neto (A paza, 2011), siendo, ademas, un estado financiero que permite ver las variaciones ocurridas en las distintas partidas patrimoniales durante un periodo económico; es decir, lo que se ha suscitado en las fuentes propias de la empresa (Giraldo, 1996). Este estado tambiên es de suma utilidad para el analista que desea descubrir el factor que determinó el cambio del monto del capital social en un período (Tanaka, 2005).

Asimismo, la NIC 1 indica que los cambios en el patrimonio de la empresa, ocurridos entre dos fechas del estado de situación financiera, reflejan el aumento 0 disminución en sus activos 0 en sus recursos propios durante el ejercicio, bajo los particulares principios de valuación adoptados y revelados en los estados financieros de dicha empresa (Flores, 2002). También norma cómo se debe presentar este estado a través de los siguientes puntos: información a revelar tanto en el estado como en las notas e información sobre las varlaciones. La entidad tiene que presentar e informar un estado de cambio en el patrimonio que muestre en la cara del estado: 1) la utilidad o perdida de periodo; 2) cada elemento de ingresos y gastos en el período que, tal y como se ha requerido por otros estándares 0 interpretaciones, sea reconocido directamente en el patrimonio y el total de esos elementos; 3) el total de Intilidas y gastos del período, calculados igual que en la ingresos y gastos del período mostrando por separado las cantidades totales atribuibles a los propietarios del patrimonio de la matriz y para los ingresos minoritarios y 4) para cada componente del patrimonio, los efectos de los cambios en las políticas de contabilidad y de las correcciones de errores reconocidos de acuerdo con la

La información a revelar de este estado en notas, es el importe de los dividendos reconocidos como distribuciones a los propietarios durante el ejercicio, y el importe por acción correspondiente (Paredes, 2009). Los cambios en el patrimonio neto de una entidad, entre el comienzo y el final del ejercicio sobre el que se informa, reflejará el incremento o disminución en sus activos netos en dicho ejercicio, excepto por lo que se refiere a los cambios que proceden de transacciones con los propietarios en su condición de tales y los costos directamente relacionados con estas transacciones, la variación global del patrimonio durante el ejercicio incluyendo el importe total de ingresos y gastos, actividades de la entidad durante el ejercicio.

La Comisión Nacional Supervisora de Empresa de $V$ alores (CONASEV) que actualmente se conoce como la SMV, esta entidad es encargada de normar la presentación de los estados financieros de las compañías que están bajo la jurisdicción (Rivero, 2006), emite un M anual para la Preparación de Información Financiera, que señala adicionalmente que, en el estado de cambios en el patrimonio, debe mostrarse separadamente 10 siguiente: 1) saldos de las partidas patrimoniales al inicio del período, 2) ganancia o perdida reconocida directamente en el patrimonio procedentes de: medición a valor razonable de inmuebles, operaciones de cobertura de flujo de efectivo y de cobertura de una inversión neta de un negocio en el extranjero y diferencia de cambio relacionadas con: ingresos, partidas monetarias y la transferencias netas de resultados no realizados $y$ otras transferencias 4) utilidad (pérdida) neta del ejercicio 5) efecto acumulado de cambios en políticas contables $y$ corrección de errores, 6) distribuciones o asignaciones efectuadas en el período de utilidades a reservas, 7) dividendos declarados y participaciones acordadas durante el período (dividendos de efectivos o en acciones y otras participaciones), 8) nuevos aportes de accionistas, 9) reducción de capital o redención de acciones de inversión, 10) acciones en tesorería, 11) capitalización de partidas patrimoniales, 12) incrementos o disminuciones por fusiones 0 escisiones, 13) conversión a moneda de presentación, 14) variación de intereses minoritarios y 4) otros incrementos o disminuciones de las partidas patrimoniales.

Saldos de las partidas patrimoniales al final del

\section{Estadb de flujos defectivo}

Otro estado financiero mencionado en la NIC 1 es el Ilamado flujo de efectivo, el cual puede ser abordado por definición e importancia.
Su conceptualización permite observar que el estado de flujo de efectivo es un estado financiero básico que, junto con el estado de resultado, proporciona información acerca de la situación financiera de un negocio, dado en un tiempo determinado y mediante (Estela, 2009). También es el estado básico que presenta la información relacionada con los recaudos y desembolsos en efectivo que se derivan de las actividades de operación, financiación e inversión, llevadas a cabo por el ente contable en un período (Rincón, 2009).

A simismo, este estado es de gran importancia porque refleja información sobre los flujos de efectivo, financieros, una base para evaluar la capacidad de la entidad para generar efectivo y equivalentes al efectivo, y las necesidades de la entidad para utilizar esos flujos de efectivo (Vera, 2007). Su objetivo principal es proporcionar información sobre los recuadros y desembolsos de dinero de la organización, con el fin de que estos puedan examinar la capacidad de la empresa para generar flujos favorables de efectivo. De igual manera, permite evaluar la capacidad de la organización para responder a sus obligaciones con proveedores, empleados, entidades financieras, accionistas o socios y demás acreedores (Sinisterra, Polanco Izquierdo, \& Henao, 2011)

Por ello, los requerimientos para la presentación de este estado, así como otras informaciones relacionadas con este estado,
Efectivo).

\section{Conclusión}

La presentación de estados financieros cumplen aspectos específicos en el ente económico como: proveer información respecto a la posición financiera, conjugar información clara y libre de errores que sirva para la toma de decisiones.

Es por ello, que la Norma Internacional de Contabilidad 1 , promueve que, mediante el cumplimiento de los aspectos tratados, permitirá a la representación financiera cumplir su importante función que es la de establecer bases para su correcta presentación, de acuerdo a la especificacion de un momento oportuno y libre de la práctica del quehacer contable en los diversos ámbitos laborales. 


\section{Referencias}

A paza, M . (2009). Las finanzas y las NIIF aplicadas a nuevo PCGE. Lima, Perú: A trium.

A paza, M. (2011). Análisis de NIIF para PYME E y casos prácticos aplicando el PCGE. Lima, Perú: Grupo A crópolis.

A paza, M . (2011). E stados financieros. Lima, Perú: Pacífico Editores.

A paza, M ., \& Estela, D. (2006). N ormas internacionales de información financiera. Lima: Entrelíneas.

A rens, Elder, \& B easley. (2007). Auditoría: Un enfoque integral (V ol. Decimoprimera edición). México, México: Pearson Educación.

A yala, P. (2003). Estado de flujos de efectivo. Lima: Pacífico Editores.

Concha, P. F. (1998). Evaluación de estados financieros. Lima: Universidad del Pacífico, Centro de Investigaciones

Effio, F. (2011). NIIF para las PYMES. Lima, Perú: Entrelíneas S.R.L.

Estela, D. (2009). Contador: aplicaciones del Plan Contable G eneralmente A ceptable. Lima: Horizonte Empresarial.

Europea, U. (6 de Junio de 2012). Reglamentos. Diario Oficial de la U nión Europes.

Ferrer, A. (2012). Estados financieros: análisis e interpretación. Lima: Pacífico Editores.

Flores Soria, J. (s.f.). Análisis e interpretación de estados financieros: teoría y práctica. Lima: Centro de Especialización en Contabilidad y Finanzas.

Flores, J. (2002). Contabilidad Nic's-Sic. M éxico: Ediciones Siglo XXI.

Flores, J. (2008). Estados financieros. Lima: Editorial Torres

García, A. (2008). A nálisis e interpretación de estados financier os. Lima: CECOF-ASESORES.

Giraldo, D. (1996). Estados financier os: teoría y práctica. Perú: San M arcos.
Glosario del M anual para la Prepación de la Información Financiera. (2008). Lima.

Glosario Tributario. (2008). Instituto de Administración Tributaria de la SUNAT (Vol. tercera Edición). Lima.

Gómez, A. (2005). Normas internacionales de información financiera. Lima: G aceta J urídica.

Gutíerrez, A . (2008). Análisis e interpretación de estados financieros. Lima: CECOF-ASESORES.

Heffes, G., Holguin, F., \& Galan, A. (1994). Auditoría de los estados finacier os: técnicas y aplicaciones modernas. M éxico: Grupo

IA SB. (2004). Normas internacionales de información financiera. España: Ciss.

IFAC. (2013). Información general sobre la IF AC. Estados Unidos.

IFRS. (2008). Normas internacionales de información financiera (NIIF). Londres: IA SCF.

J aime Flores Soria. (2008). Estados financieros: nuevo manual para la preparación de información financiera. Lima: Editorial Real Time.

J uárez, L. (2011). Principiios de contabilidad: enfoque emprendedor. M éxico: CENGA GE Learning.

J unta de Decanos de Colegios de Contadores Públicos del Perú. (2008). Manual internacional de pronunciamientos de auditoria y aseguramiento. Lima, Perú: IFAC.

K ennedy, R., \& M cM ullen, S. (1978). Estados financier os: forma, análisis e interpretación. M éxico: Editorial U teha.

Macías, R. (2011). Estados financieros: teoría y práctica. Lima: Entrelíneas.

M antilla, S. (2011). Estándares/Normas internacionales de información financiera (IF RS/NIIF) (Vol. Segunda Edición). Colombia: ECOE EDICIONES
Martínez, A. (2010). Consolidación de estados financieros: contabilidad avanzada para grupos de empresas (V ol. $3^{\text {a }}$ edición). (J. M. Chacón, Ed) México: Mc G raw Hill.

Martínez, A. (2011). Consolidación de estados financieros ( $V$ ol. za edicicón). M éxico: Litográfica Ingramex.

M eigs, Willims, Hka y Bettner. (2000). Contabilidad: I base para decisiones ( $\mathrm{V}$ ol. $11^{\mathrm{ma}}$ Edición). Colombia: Editora: Emma A riza Herrera.

Morrisey, L. (1977). Teoría contable de la información financiera. M éxico: Trillas.

Ortega, R., \& Luján, L. (2006). M odificaciones a la normativa contable. Lima: Informativo Caball ero Bustamante.

Ortega, S., Pacherres, A ., \& Díaz, R. (2011). Informativo caballero bustamante. Informativo Caballero Bustamante.

Paredes, C. (2 de diciembre de 2007). Cambio en la NIC 1: Presentación de estados financieros. Actualidad Empresarial (149), VII - 1 .

Paredes, C. (15 de diciembre de 2008). Nueva norma internacional de contabilidad NIC 1 . Presentación de estados financieros. Actualidad Empresarial(173), VI-5.

Paredes, C. (diciembre de 2008). Nueva norma internacional de contabilidad NIC 1-

Presentación de estados financieros. Actualida Empresarial, VI-5.

Paredes, C. (15 de enero de 2009). Nueva norma internacional de contabilidad NIC 1 Presentación de estados financieros. Actualidad Empresarial, III-4.

Paredes, C., \& A yala. (2009). Normas internacionales de contabilidad NIIF : interpretaciones SIC's Y CINIIF's (V ol. Tomo I). Lima: Editores Pacífico.

Paredes, Isaías V era. (s.f.). Normas internacionales de información financiera-Tomo I. Informativo Vera Paredes.

aredes Reátegui Carlos. (2009). Nueva norma internacional de contabilidad - Presentacióm de estados financieros (Parte II). Actualidad Empresarial N. ${ }^{\circ} 174, \mathrm{III}-4$.

Ralph, \& Stewart (1971). Estados financieros: forma análisis e interpretación. M éxico, M éxico: U.T.E.H.A.

Rincón, C. (2009). Contabilidad siglo XXI. B ogotá: ECOE Ediciones.

Rivero, E. (2006). Cotabilidad I. Lima: Universidad de Pacífico.

Rojas, L. (2011). F undamentos de análisis de estados financieros. M éxico: Instituto M exicano de Contadores Públicos.

Romero, Á. (2010). Principios de contabilidad (Vol. 4 a edición). México: Mc Graw Hill.

Samuel A lberto M antilla. (2006). Estándares normas internacionales de información financier (IF RS/NIF). B ogotá: ECOE Ediciones.

Sinisterra, G., Polanco Izquierdo, L. E., \& Henao, H. (2011). Contabilidad: sistema de información para las organizaciones. Bogotá, Colombia: M C Graw Hill Educación.

Superintendencia de M ercado de V alores. (2008). SM V

Tanaka, G. (2005). Análisis de estados financieros para la toma de decisiones. Lima: Fondo Editorial de la Pontificia Universidad Católica del Perú.

Tribunal Constitucional del Perú. (1993). Constitución Política del Perú. Lima, Perú.

Unión Europea. (28 de marzo de 2013). Diario oficial de la Unión Europea. Diario oficial de la Unión Europea, 81-82.

V era, I. (2007). Normas internacionales de información financiera (antes NIC's). Lima: Informativo $\checkmark$ era Paredes.

Zavala, P. A. (2003). Estado de flujos de efectivo. Lima: Pacífico Editores. 